\title{
"Gravitationally Lensed Gravitation" (GLG)
}

\author{
A Gedankenexperiment provides evidence for the existence of \\ a previously overlooked effect in General Relativity
}

Andreas Boenke (e-mail: Scilencium@gmx.net)

\begin{abstract}
The intention of this paper is to point out a remarkable hitherto unknown effect of General Relativity. Starting from fundamental physical principles and phenomena arising from general relativity, it is demonstrated by a simple Gedankenexperiment that a gravitational lens enhances not only the light intensity of a background object but also its gravitational field strength by the same factor. Thus, multiple images generated by a gravitational lens are not just optical illusions, they also have a gravitational effect at the location of the observer !

The "Gravitationally Lensed Gravitation" (GLG) may help to better understand the rotation curves of galaxies since it leads to an enhancement of the gravitational interactions of the stars.

Furthermore, it is revealed that besides a redshift of the light of far distant objects, the cosmic expansion also causes a corresponding weakening of their gravitational effects.

The explanations are presented entirely without metric representation and tensor formalism. Instead, the behavior of light is used to indicate the effect of spacetime curvature. The gravitation is described by the field strength which is identical to the free fall acceleration. The new results thus obtained provide a reference for future numerical calculations based on the Einstein field equations.
\end{abstract}

\section{Introduction and Historical Overview}

More than a hundred years ago, the astronomer Sir Arthur Stanley Eddington successfully measured the deflection of light in the gravitational field of the Sun for the first time. In so doing he impressively confirmed the prediction of Einstein and initiated the breakthrough of the General Relativity theory. During the total eclipse which occurred on May $29^{\text {th }} 1919$ on the Island of Principe he took photographs of a group of bright stars near the periphery of the darkened Sun. A comparison with photographs of the same stars in the night sky taken half a year earlier revealed the value predicted by Einstein for the apparent shift in star positions. It is particularly entertaining to read in [1] how the historical circumstances lead to the perfect timing, so that the measurement of the gravitational light deflection only succeeded after Einstein had correctly calculated the size of the effect with his field equations.

Irrespective of this early triumphal confirmation of his theory by accurate astronomical observations Einstein did not believe that it would ever be possible to observe real lensing by gravitational fields. What is meant by this is not just a tiny shifting of apparent star positions but a veritable focusing of light rays of a source and the resulting enhancement of its apparent brightness at the location of this focus. Only at the insistence of the engineer Rudi W. Mandl he published a brief article about the functional principle of such gravitational lenses in 1936 [2]. The obvious irrelevance of the subject to him was probably the reason why he did not deem it worth further considerations. 
Astronomers were not so pessimistic. Fritz Zwicky immediately took up the subject and saw galaxies and galaxy clusters as suitable candidates for such gravitational lenses. Already in 1937, he proposed to look for such natural lenses and to use their magnification for observation of extremely far background objects which would otherwise be out of reach of our telescopes. Furthermore, the gravitational lens effect could help to determine the mass of the galaxy causing it [3].

Two and a half decades later Sjur Refsdal thought through the details of the gravitational lens effect. He found methods which, besides angular distances, also use differences of light travel times of two images in order to determine the lens galaxy's mass and also the Hubble Constant. In 1964 he proposed to search for a supernova in a background galaxy behind a gravitational lens which would then appear at different times in the images of that background galaxy [4].

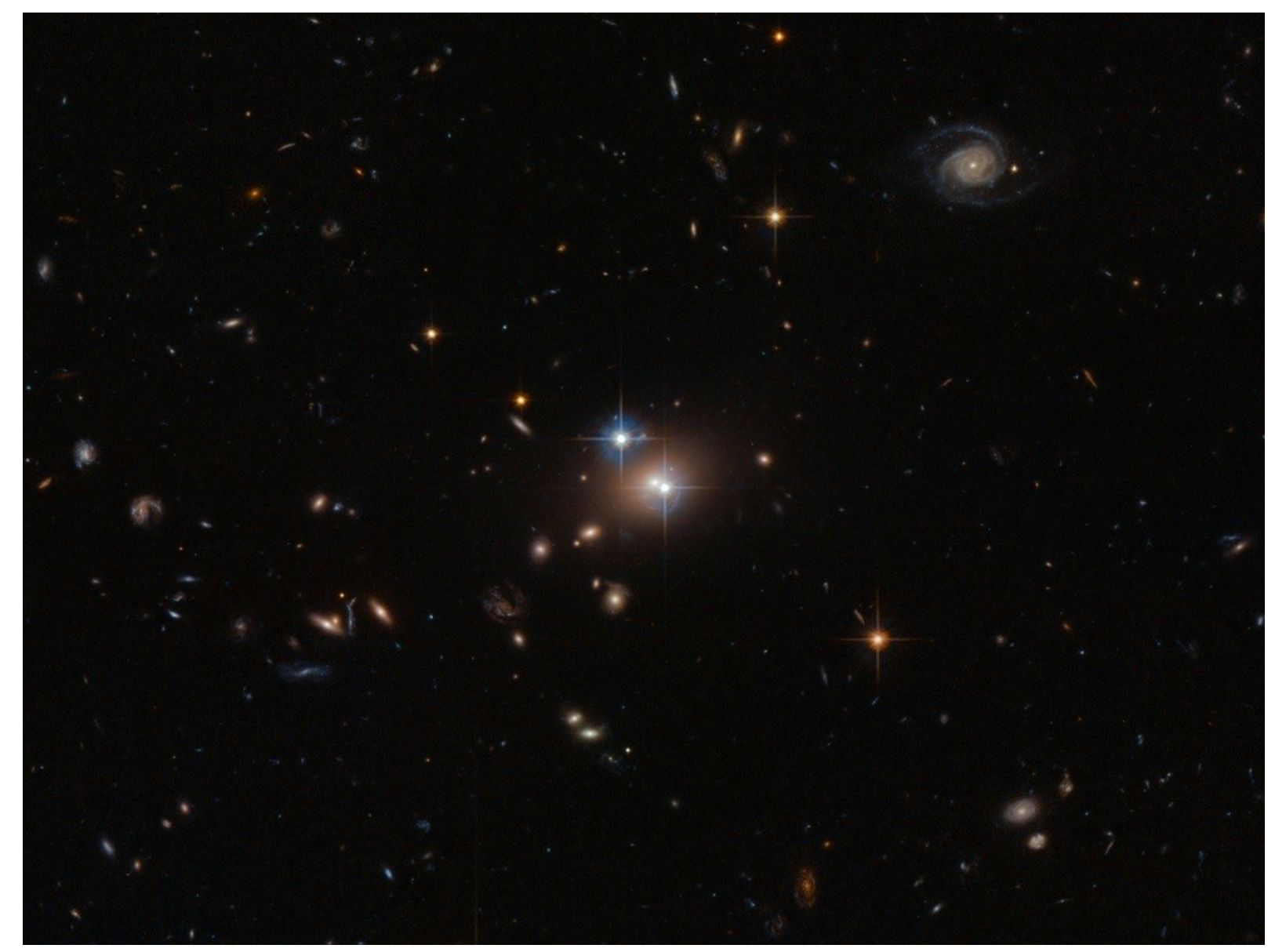

Fig. 1: The "Twin Quasar" QSO 0957+561A/B in the Constellation Ursa Major. Credit: ESA/Hubble \& NASA [5]

After this theoretical groundwork it took another 15 years until the first gravitational lens was found. In early 1979 the astronomers Dennis Walsh, Robert Carswell and Ray Weymann discovered, with the 2,1meter-telescope at Kitt-Peak in Arizona, two objects today known as "Twin Quasar" (see center of Fig. 1: two bright "stars" with a bluish cross of rays beside a yellowish diffuse galaxy). Since the two quasars seem to be unusually close to each other and show an identical redshift the astronomers realized soon that these are two images of the same object, generated by a gravitational lens in the foreground [6] [7]. Sjur Refsdal too was engaged with a group of his own in the subsequent investigations. The common origin of both images could be confirmed by recording their brightness curves during many years of observation. It turned out that the light of the upper left image reaches the Earth about 14 months earlier than the light 
of the lower right image. So, we see the source not only from two slightly different perspectives but we also see it at two different points of time of its past !

30 years ago the mission of the Hubble Space Telescope started which has refined and enriched our vision of the universe by a multitude of spectacular detailed pictures. Among these are pictures in which multiple images of background galaxies are visible, partly distorted to arcs or rings, which can be attributed to the effect of gravitational lenses in the foreground. With the aid of the Hubble Space Telescope astronomers in 2014 actually succeeded to find a supernova in a background galaxy which is imaged by a gravitational lens multiple times. The detailed analysis of the gravitational lens and the light travel times of the individual images culminated in a correct prediction of the supernova event in one of the images. In memory of the great pioneer in the research field of gravitational lensing the supernova was named "SN Refsdal" [8].

Last but not least also this work was inspired by the Hubble Space Telescope. While looking at one of its wonderful photographs, it suddenly became clear to me what the images of a gravitational lens imply. The center of Fig. 1 shows two images of a quasar far behind a foreground galaxy which acts as a gravitational lens. Heading towards the upper left image and travelling in imagination until one reaches a position beside the foreground galaxy, one will have a free view of the quasar located further beyond. This works similarly with the lower right image, except that one will have a slightly different perspective onto the quasar. On both paths there is no obstacle for the light, nothing on which it is refracted. It propagates freely through the curved spacetime, whose curvature is caused by the gravity of the foreground galaxy. And exactly the same applies to all effects which propagate at the speed of light. Images generated by gravitational lenses, often referred to as "mirage", are far more than just optical illusions !

It is widely known that all energy flows propagating at the speed of light (photons, neutrinos and gravitational waves) follow the same paths and are "deflected" in gravitational fields in exactly the same way as light.

It is hardly known, however, that this also applies to the electric field of a point charge at rest in a gravitational field. Electric field lines are following exactly the same paths as light rays originating from the same point [9].

It is yet unknown that this applies quite analogously even to gravitational fields. As much as a gravitational lens enhances the light intensity which reaches the observer from a background object, it also enhances the gravitational effect due to that object. The naïve notion that where we see two quasars we experience the gravitational effect of two quasars is correct, even if we later learn from detailed analysis that we are dealing with two images of a single quasar !

That it has to be that way will be shown below with the "Gravitational Lens Gedankenexperiment", which we will approach in three steps. The first step is to introduce the scenario of a sudden light burst event of a star and to derive fundamental relations between light and gravitational fields. These will then be used to conclude the behavior of gravitational fields from the behavior of light. The second step is to illustrate the experiences of an observer witnessing three such events whose effects are superimposed. In the third step we will finally come to the actual thought experiment and the surprisingly simple conclusion from it - that light and gravitation are affected by spacetime curvature in the same way. 


\section{Step One: Introduction of the scenario of a sudden mass loss during a light burst event}

We consider a star which undergoes a sudden mass loss by isotropic emission of the equivalent energy amount throughout a light burst.
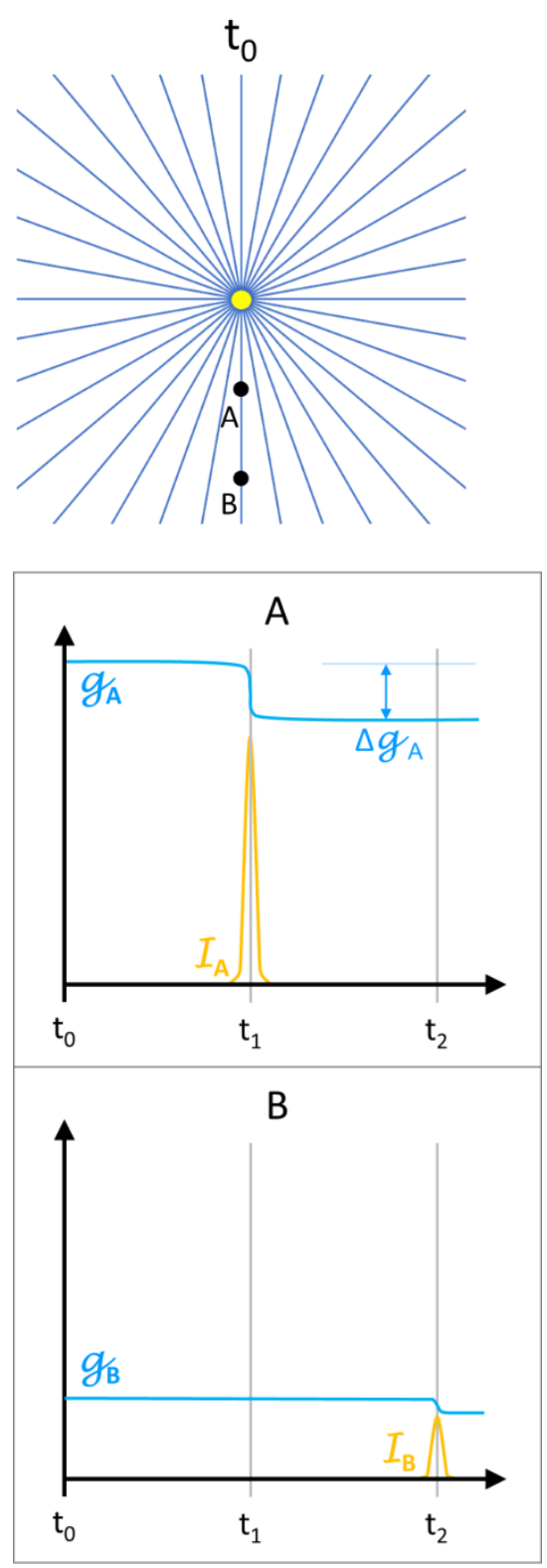
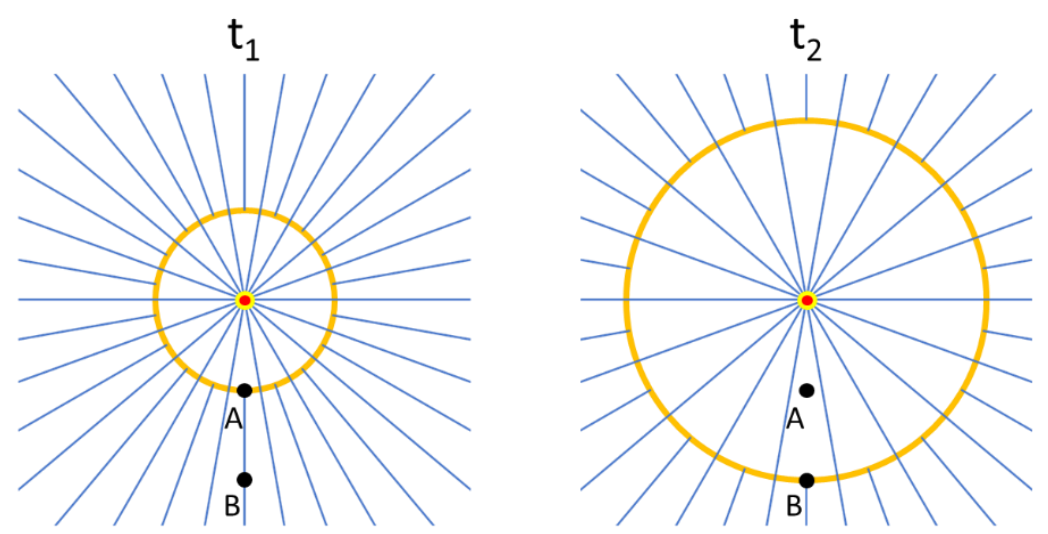

Fig. 2: Above: Isotropic expansion of a shell of photons (orange) generated throughout a light burst event, depicted at three successive moments in time. The radial blue lines represent field lines of the gravitational field.

Left: Diagrams of the temporal development of the intensity l of the light pulse (orange) and of the gravitational field strength $g$ (blue) as perceived by observer $A$ and $B$ (both diagrams have the same scale).

Depicted above is the situation at three consecutive moments with the star in the center and the observers "Alice" (A) and "Bob" (B) resting at different distances from the star. The orange circle represents the expanding spherical shell of the photons from the light burst. At the time $t_{0}$ the light burst event occurs, at $t_{1}$ the shell of photons reaches observer $\mathrm{A}$, at $\mathrm{t}_{2}$ it reaches observer $\mathrm{B}$ at $\mathrm{a}$ greater distance from the star. For all the considerations following below we assume weak gravitational fields.

As long as the shell of light energy (the photons) has not reached an observer he (or she) experiences the unchanged gravitational field strength = gravitational acceleration $g$. When the shell of photons passes by, he measures a narrow-peaked intensity pulse. At the same time the gravitational field strength is reduced by $\Delta g$. These ideas are based on the following principles:

\section{Birkhoff's theorem:}

The gravitational field outside a spherical symmetric expanding shell of emitted energy stays unchanged, whereas inside the shell it is determined only by the reduced residual mass $m$ * of the star remaining in the center (see e.g. [10]). This statement combines the following consequences of causality and consistency. 


\section{Causality:}

The sudden decline of the gravitational field strength carries information about the sudden mass reduction of the star. This information cannot propagate faster than the light.

\section{Consistency:}

The sudden decline of the gravitational field strength constitutes a monopole-like disturbance of the gravitational field, which according to the Einstein field equations cannot propagate freely. It remains bound to the expanding shell of light energy, so it cannot propagate slower than the light.

What is really impressive is the animation in [11]. Here Claudia de Rahm lets the entire Sun simply disappear. According to the Einstein field equations such monopole type changes of the gravitational field cannot propagate freely. They remain bound to the expanding shell of energy (photons, neutrinos or gravitational waves), which can be read in [12], in [13] and in [14].

\section{Basic Relations}

By isotropic emission of energy throughout a light burst event a star suddenly reduces its mass about $\Delta m$, which equals the fraction $\eta$ of its mass $m$ before the burst:

$$
\eta=\frac{\Delta m}{m}
$$

$\eta$ is the efficiency of the conversion of mass into light energy during the burst event. The initial mass $m$ of the star can be differentiated into the residual mass $m^{*}$ staying in place and the mass $\Delta m$ leaving as light:

$$
m=m^{*}+\Delta m
$$

Before the arrival of the light pulse at an observer, the gravitational field contributions of these mass fractions are adding up there. For the weak field far away from the single star we can use the approximation by Newtons law of gravity ("Newtonian limit"). At the distance $r$ from the center of the star the field strength $g$ (which equals the free fall acceleration of an object previously at rest) is given by:

$$
g=\frac{G}{r^{2}} \cdot m=\frac{G}{r^{2}} \cdot\left(m^{*}+\Delta m\right)=g^{*}+\Delta g
$$

$G$ is the gravitational constant. At the time when the shell of propagating energy passes an observer, the contribution to the gravitational field due to the mass $\Delta m$ vanishes. The absolute reduction of the field strength $\Delta g$ is:

$$
\Delta g=\frac{G}{r^{2}} \cdot \Delta m
$$

Furthermore, every observer claims that the relative reduction of the gravitational field strength equals the fraction $\eta$ by which the mass of the star was reduced:

$$
\frac{\Delta g}{g}=\frac{\Delta m}{m}=\eta
$$

This follows from (3) and (4). So, the initial gravitational field strength $g$ can be calculated from the absolute reduction $\Delta g$ if the value $\eta$ is known:

$$
g=\frac{\Delta g}{\eta}
$$


According to the most famous formula of Special Relativity the total energy $E$ of the light burst is:

$$
E=\Delta m \cdot c^{2}
$$

The assumed isotropy means that the energy $E$ of the photons of the light burst is evenly distributed over the expanding spherical shell with (continuously increasing) radius $r$ and surface $A$. The energy per perpendicularly irradiated area is the radiant exposure $H$, henceforth shortly referred to as "exposure". $H$ can be written as:

$$
H=\frac{E}{A}=\frac{\Delta m \cdot c^{2}}{4 \pi \cdot r^{2}}
$$

Rearranging (8) to get an expression for $\Delta m$ and inserting this into equation (4) leads to the cancellation of $r$ because the exposure $H$ and the field strength $g$ have the same dependency on the distance $r$.

The result is the universal relation:

$$
\Delta g=\frac{4 \pi \cdot G}{c^{2}} \cdot H
$$

The local absolute reduction of the field strength $\Delta g$ has always and everywhere the same constant ratio to the local exposure $H$ from the light burst, independent of the specific "history" of its generation, so also independent of its distance $r$ from a certain star. For example, quadrupling the mass $\Delta m$ together with doubling the distance $r$ leads to the same findings of the observer. Such scenarios are indistinguishable to him ! The energy density locally passing by the observer solely determines - or more precisely: causes the local change of the gravitational field strength or acceleration. From the consideration of a concrete simple scenario with well-known identical global position dependency of field strength and exposure we have derived this local connection, which we consider as valid everywhere in the universe and will use below as a "tool" for analyzing more complex scenarios hereinafter.

From (9) together with (6) follows:

$$
g=\frac{4 \pi \cdot G}{\eta \cdot c^{2}} \cdot H
$$

The value of $\eta$ is a constant which only depends on the specific burst event of the source.

$$
\frac{g}{H}=\frac{4 \pi \cdot G}{\eta \cdot c^{2}}=\text { const. }
$$

The position-dependent properties of the gravitational field strength $g$ and the exposure $H$ originating from the same source have the same constant ratio everywhere. That means they show the same position dependencies!

We applied Birkhoff's theorem of General Relativity in the Newtonian limit to the simple scenario of a single star, which undergoes a sudden mass loss by isotropic emission of a light burst. This led to the discovery of the universal relation (9) between the local change of the gravitational field strength and the local areal energy density passing by. Using that relation, the identical global position dependency of field strength and exposure was derived. This is the correct result for our example of a single star, but it is generally valid.

These simple relations provide the base for our further considerations with stunning results and far-reaching consequences. 
Remarks:

A few more words about the connection between "exposure" and the more common property "intensity". The intensity $I$ is defined as radiant power $P$ per perpendicularly irradiated area $A$ :

$$
I(t)=\frac{P(t)}{A}
$$

The temporal curve of the light intensity $I(t)$ varies strongly during the light burst. It has the shape of a narrow-peaked pulse. The time integral of the radiant power $P(t)$ over the pulse duration gives the total energy $E$ to which the area $A$ was exposed:

$$
E=\int P(t) d t
$$

The exposure $H$, which is the energy per area, can also be written as:

$$
H=\frac{E}{A}=\frac{1}{A} \int P(t) d t=\int \frac{P(t)}{A} d t=\int I(t) d t
$$

So it turns out that the exposure is just the time integral of the intensity over the duration of the light pulse. This corresponds to the areas under the temporal intensity curves of the light pulses in the diagrams. 
Step Two: Linear superposition of the effects of three similar events

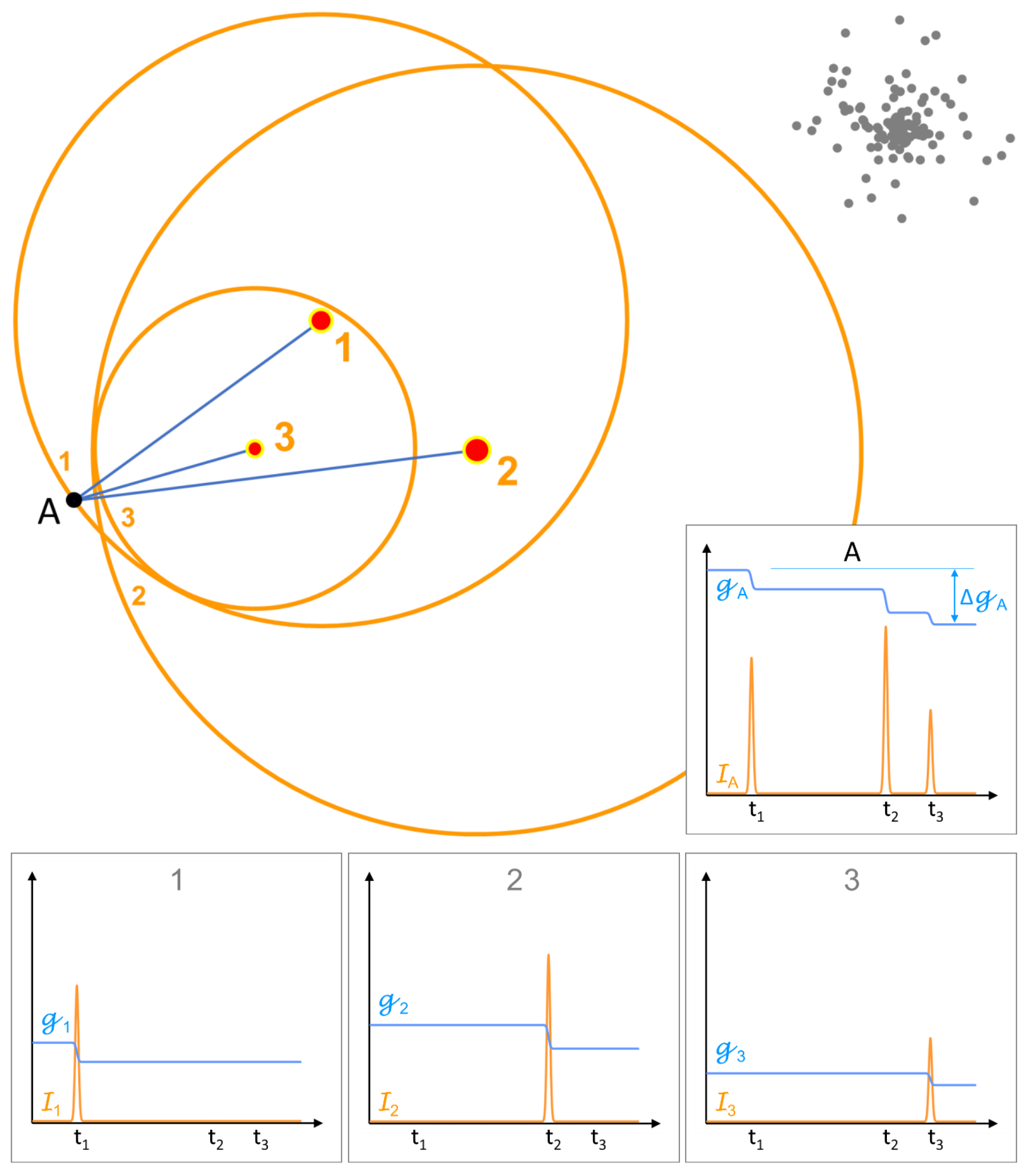

Fig. 3: Superposition of the effects of three different stars undergoing similar light burst events. Depicted is the situation at the time $t_{1}$, when the light pulse from star 1 arrives at the observer $A$. The diagrams 1,2 and 3 show the temporal development of the gravitational field strength $g$ (blue) and of the light intensity I (orange) at the position of observer $A$ as she would perceive if only star 1, 2 or 3 existed alone. So, these are the individual contributions which add up to the effective total values $g_{A}$ and $I_{A}$ as shown in diagram $A$.

Now we extend the scenario to an arrangement of three individual stars. One after the other meets a similar fate as our lone star described above. 
"Similar" means that each star is supposed to lose the same fraction $\eta$ of its individual mass during its light burst event. This special assumption seems to come out of the blue but its usefulness will become clear soon.

Due to the different masses of the stars and their different distances the light pulses arriving at the observer "Alice" (A) one after the other have different peak intensities. Together with each light pulse a step-wise reduction of the gravitational field strength occurs, which is proportional to the exposure from that individual light pulse. Because of the assumed "similarity" of the light burst events each of these step-wise reductions $\Delta g_{i}$ represents the same fraction $\eta$ of the previous contribution $g_{i}$ of the corresponding star:

$$
\frac{\Delta g_{1}}{g_{1}}=\frac{\Delta g_{2}}{g_{2}}=\frac{\Delta g_{3}}{g_{3}}=\eta
$$

The total reduction $\Delta g_{A}$ across the three steps is thus the fraction $\eta$ of the total initial gravitational field strength $g_{A}$ :

$$
\Delta g_{A}=\Delta g_{1}+\Delta g_{2}+\Delta g_{3}=\eta \cdot\left(g_{1}+g_{2}+g_{3}\right)=\eta \cdot g_{A}
$$

Unsurprising: The three light pulses together with the three steps of reduction of the gravitational field strength lead to the conclusion that there are three individual contributions to the gravitational field which are superimposed so that the total gravitational field is stronger than each individual contribution alone.

\section{Remarks:}

Here we are especially interested in the contributions of the three stars to the total gravitational field strength and the reduction of these contributions due to the light bursts. A contribution of the background galaxy (gray) is not considered any further since it represents only a constant offset.

Besides that, we assume small angles between the lines of sight from the observer A to the three stars, so that we can simply sum up their contributions to the gravitational field strength instead of having to vectorially add them. 


\section{Step Three: The "Gravitational Lens Gedankenexperiment"}

Finally, we examine a gravitational lens in detail by applying the relations introduced above. Fig. 4 is based upon a drawing (Fig. 4 in [15]) of Sjur Refsdal, whose work was groundbreaking for the exploration and understanding of gravitational lenses.

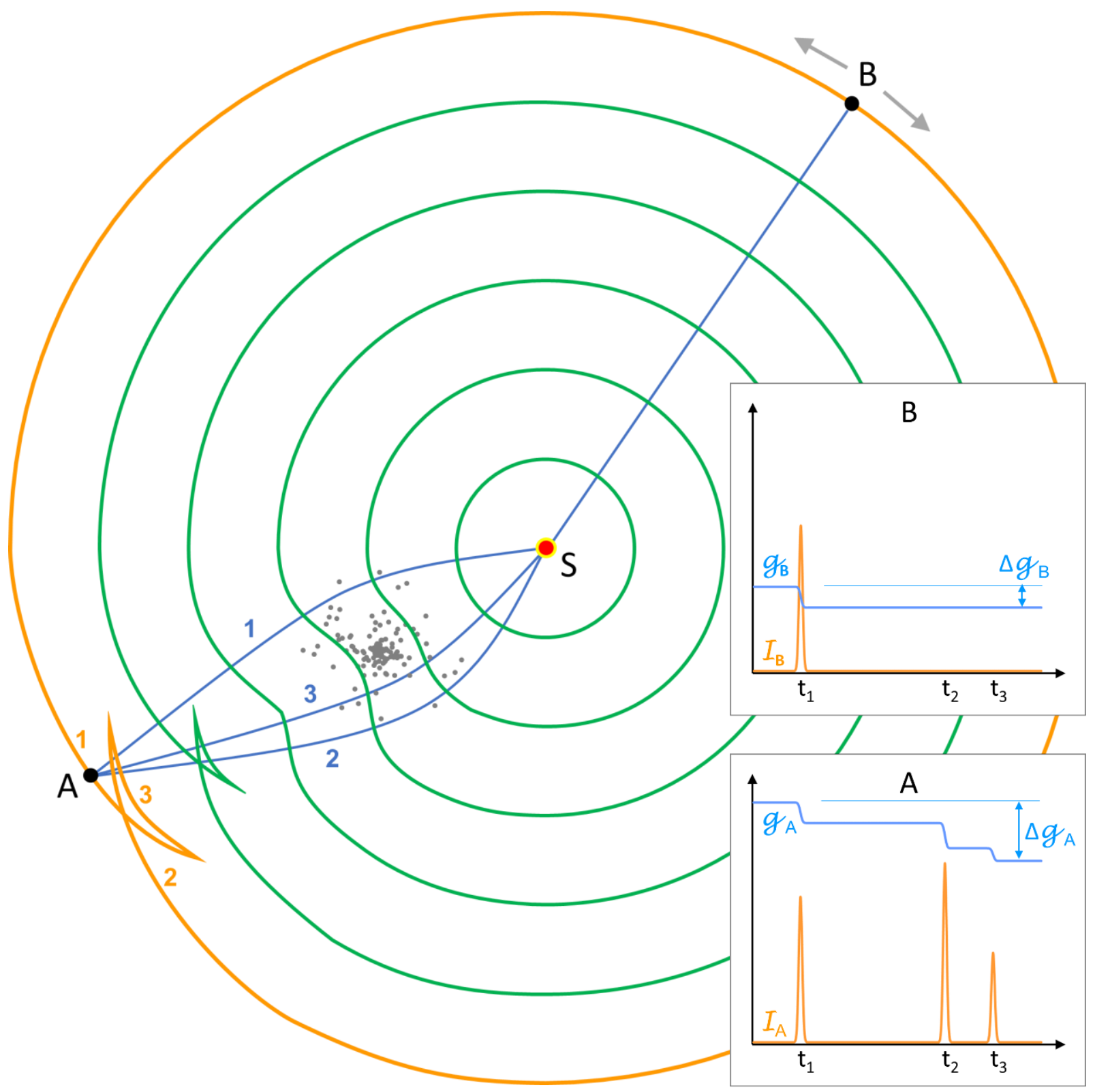

Fig. 4: The wavefronts (green) of light emitted by the source $S$ are delayed while passing through the gravitational field of a mass assembly (gray). In their further course they are deformed and folded. This corresponds to a curvature of the paths of photons (blue). Light from the source arrives at observer $A$ from several directions, so that $A$ sees several images of the source in these directions. To the observer $B$ the light is not affected and its path is a single (almost) straight line.

The different travel times of the light on the individual curved paths mean that photons emitted by source $S$ at once arrive at the observer "Alice" (A) at different times. To put it the other way round, this means that the individual images which the observer A simultaneously sees consist of photons emitted at different times. So the different images show the source $S$ at different times of its past. 
However, on its way to observer "Bob" (B) the light is not affected by a gravitational lens. As usual, this observer sees just one source (one image) in its past state at the emission time of the light.

For the thought experiment we now put our star at position $\mathrm{S}$ and let it constantly shine for a long time before the light burst occurs. The gravitational lens provokes three images of the constantly shining star at observer A which have different intensities. It is supposed that the total intensity of all three images is higher than the intensity of the single image which observer B receives:

$$
I_{1}+I_{2}+I_{3}=I_{A}>I_{B}
$$

The ratio $v$ of the intensities can be denoted as "enhancement" by the gravitational lens:

$$
\frac{I_{A}}{I_{B}}=v
$$

According to the above assumption we have $v>1$ here.

The constant intensities of the images before the light pulses cannot be seen in the diagrams above. They are several orders of magnitude smaller than the peak intensities of the light pulses and cannot be distinguished from the zero value in the linear scale. The intensities of the images are determined by the individual curved paths from the star to the observer through the gravitational lens. These paths are the same for the light emitted throughout the light burst event. Thus, for an individual image the peak intensity of the light pulse - and with this also the exposure $H$ - is proportional to the previous constant intensity $I$ of that image. A brighter image will also show a brighter light pulse. This proportionality applies to every image and any observer, since all images are derived from the same object, namely our star:

$$
\frac{H_{1}}{I_{1}}=\frac{H_{2}}{I_{2}}=\frac{H_{3}}{I_{3}}=\frac{H_{B}}{I_{B}}
$$

After the light burst event of our star the gravitational lens induces the folding of the expanding shell of photons (orange) as shown in Fig. 4. Each time that a section of the folded shell of photons passes the observer A she notices a steep increase, a peak and a steep decrease of the intensity of the corresponding image. Hence three such light pulses arrive at her one after the other. For the total exposure $H_{A}$ it follows from (19):

$$
\frac{H_{1}+H_{2}+H_{3}}{I_{1}+I_{2}+I_{3}}=\frac{H_{A}}{I_{A}}=\frac{H_{B}}{I_{B}}
$$

This can be rearranged to:

$$
\frac{H_{A}}{H_{B}}=\frac{I_{A}}{I_{B}}=v
$$

Now we wonder what the sudden mass reduction of our star during the light burst event means for its gravitational effect at observer A and B. Let's start with observer B. The shell of photons propagating from the star to him is not affected by the gravitational lens, so he sees just a single light pulse with exposure $H_{B}$ and at the same time notices a proportional single reduction of the gravitational field strength $\Delta g_{B}$. We recall the universal relation derived above (9) between the local reduction of the gravitational field strength and the exposure from the light pulses:

$$
\Delta g_{B}=\frac{4 \pi \cdot G}{c^{2}} \cdot H_{B}
$$


With the known fraction $\eta$ of the mass reduction we can use equation (6) to calculate the initial contribution $g_{B}$ of our star to the gravitational field strength before the arrival of the light pulse:

$$
g_{B}=\frac{\Delta g_{B}}{\eta}
$$

The experience of observer A is quite different. There the gravitational lens itself causes the main contribution to the gravitational field, but this is constant and is not considered any more. We are especially interested in the contribution of our star, which alone is responsible for the reduction of the gravitational field.

The gravitational lens is the cause why observer A sees three images of our constant shining star and then perceives three times a light pulse together with a reduction of the gravitational field. With (9) we get:

$$
\Delta g_{A}=\Delta g_{1}+\Delta g_{2}+\Delta g_{3}=\frac{4 \pi \cdot G}{c^{2}} \cdot\left(H_{1}+H_{2}+H_{3}\right)=\frac{4 \pi \cdot G}{c^{2}} \cdot H_{A}
$$

Concerning the contribution of our star to the gravitational field strength: Its overall reduction must represent the same fraction $\eta$ of its initial value as the mass reduction does. So, the same relation as (16) results:

$$
\Delta g_{A}=\Delta g_{1}+\Delta g_{2}+\Delta g_{3}=\eta \cdot\left(g_{1}+g_{2}+g_{3}\right)=\eta \cdot g_{A}
$$

Astonishing: The three light pulses together with the three steps of reduction of the gravitational field strength lead to the conclusion that there are three individual contributions to the gravitational field which are superimposed so that the total gravitational field is stronger than each individual contribution alone.

The local effects of the three images of our single star generated by the gravitational lens cannot be distinguished by observer A from the local effects of three individual stars undergoing a similar fate. "Alice can make accurate predictions by judging a situation on the basis of what she actually sees. I call this the seeing-is-believing principle." Marek Artur Abramowicz describes this basic principle in a different context [16], but it also holds true here.

All the star images generated by the gravitational lens are not just optical illusions but also have gravitational effects upon the observer !

From the above assumption $v>1$ we obtain the following result: At observer A the total exposure $H_{A}$ from the three light pulses is higher than $H_{B}$ at observer B. Correspondingly the overall reduction of the gravitational field strength $\Delta g_{A}$ is greater than $\Delta g_{B}$. Since the overall reduction of the gravitational field strength is caused by one and the same light burst event of our star, it represents the same fraction $\eta$ of the initial gravitational field strength everywhere. This fraction equals the ratio between mass reduction and initial mass of our star. Thus the initial gravitational field strength $g_{A}$ at observer A was higher than $g_{B}$ at observer B as well!

$$
g_{A}=\frac{\Delta g_{A}}{\eta}
$$

Putting all this together we always get the same ratio:

$$
\frac{g_{A}}{g_{B}}=\frac{\Delta g_{A}}{\Delta g_{B}}=\frac{H_{A}}{H_{B}}=\frac{I_{A}}{I_{B}}=v
$$

At the position of observer A, the gravitational lens not only enhances the light intensity but also the gravitational field strength originated from our star! 


\section{Continuity}

If one imagines the position of observer B to be continuously shifted along the shell of photons towards the position of observer A, he will arrive counter-clockwise at section 1 and clockwise at section 2. During this continuous shifting the properties of the shell of photons will also only change continuously between neighboring temporary positions of observer B. Even where a position is reached that other sections of the folded shell of photons will pass by later on, no abrupt changes are expected. From Fig. 4 it becomes clear that there are also positions where section 2 arrives before section 1 . These sections have to be regarded as connatural and independent of one another, as if originated from different sources.

\section{As the light, so the gravity: The analogy between field lines and light rays}

The above derived equality of the ratios (27) between gravitational field strengths and intensities at the positions of two observers A and B can be rearranged:

$$
\frac{g_{A}}{I_{A}}=\frac{g_{B}}{I_{B}}=\text { const }
$$

Now we see that the ratio between the gravitational field strength contribution and the light intensity, both originating from our constant shining star, has the same constant value everywhere. This value is only dependent of the mass and the total radiant power of our star. It is not position-dependent and has the same value everywhere within the range of influence of our star. Especially the presence of the gravitational lens does not change this ratio because the curvature of spacetime affects both the gravitational field and the light from our star in the same way. Within the model of light rays the local intensity and the local exposure are proportional to the density of light rays per perpendicularly irradiated area. Within the model of field lines, the field strength is likewise proportional to the density of field lines per perpendicularly crossed area. The constant position-independent ratio between gravitational field strength and light intensity requires identical paths of field lines and light rays originating from our star. The gravitational lens is a curvature of spacetime which effects a transversal curvature of the light rays from our star. This leads to an increased light intensity at observer A. At the same time, it effects the identical curvature of the field lines from our star which leads to an identical increase of the gravitational field strength at observer A.

I termed this effect of distortion of gravitational fields by gravitational lenses as

"Gravitationally Lensed Gravitation" (GLG)

or "gravity-lensed gravity" for short. As far as I know, it was not described anywhere else before.

Here again the chain of reasoning without the notion of field lines which is frowned upon in the community of experts in General Relativity:

A gravitational lens generates three images of a constant shining star and thus causes an enhancement of the total light intensity from that star at observer A. Likewise it causes an equal enhancement of the total exposure from the light pulse arriving three times at observer A. The associated reduction of the gravitational field strength over all three steps is equally enlarged. This total reduction represents a certain fraction of the initial gravitational field strength of the constant shining star. So the latter must have been equally enhanced as the light intensity !

$$
\frac{g_{A}}{g_{B}}=\frac{I_{A}}{I_{B}}=v
$$


Observed enhancements of light intensities by gravitational lenses range from several times (the microlensing effect of single stars) up to a thousand times (the macrolensing effect of galaxy clusters). In view of this a considerable relevance of the GLG effect for astrophysics cannot be denied, since the enhancements of the gravitational fields of the background objects will be of equal amounts.

In the configuration of the Gedankenexperiment described here, light is exemplarily used to make visible the effect of spacetime curvature on any and all interactions which propagate at the speed of light. In this connection any attenuation of the light due to absorption or scattering is neglected. Observed enhancements of light intensities may be reduced as a consequence of such attenuations. This likely is the case for the third image in the Twin Quasar (Fig. 1) too, which should exist according to Fig. 4. Its absence is explained with attenuation and is further investigated (e.g. [17]). Thus, observed enhancements of light intensities represent only lower estimates for the enhancements of gravitational fields, which do not undergo any attenuation when penetrating matter. Naturally the enhancement of gravitational fields is the same for all objects, no matter whether they shine or not. Instead of light as indicator for the effects of spacetime curvature we could have taken neutrinos just as well. Doing so we would not have to worry about attenuation.

Of course, the gravitational field will not be enhanced in all directions. Let us again recall the effects on light. The gravitational lens just causes a curvature of the paths of the photons and does not generate additional photons. It is just a redistribution of the light that takes place. The intensity is therefore increased in some places and reduced in others. Correspondingly, the field lines are only redistributed by the curvature, concentrated at some places, where the field strength increases, thinned out at other places, where the field strength decreases. Here again I take advantage of the comprehensible field line model since it considerably simplifies the description of the relations. Flexibly thinking theorists are welcome to perform the translation into the language of metric tensors.

\section{Remarks:}

The concept of curved field lines following the path of photons (null-geodesics) may also have a very practical use. The representation of gravitational fields with such "geodesic field lines" may help to significantly simplify numerical calculations in General Relativity. Methods for computation of the intensity of light in caustics of gravitational lenses are well known and proven. In certain cases, the results can be used without any further changes and just have to be reinterpreted in terms of gravitational field strength contributions.

The GLG claims the same analogy between field lines and light rays as Thanu Padmanabhan already found for the electric field (see [9]). These analogies can be derived more directly from the equivalence principle of General Relativity by interpreting light rays as field lines of a radiation pressure field, which is chosen so that it just compensates an electric or gravitational field of a point source.

The strict locality and the equivalence of the subjective perspectives of all observers are essential elements of Einstein's theories of Relativity. The appropriate principle "seeing-is-believing" (M. A. Abramowicz [16]) is fulfilled by the GLG: Where the observer sees three objects, she also experiences the gravitational fields of three objects. From the instantaneous local effects, the observer cannot distinguish the three images generated by the gravitational lens from three individual existing stars. Needless to say, the three images of our star will show a different long-term behavior than three independently existing stars which will move individually and interact with each other or with their surroundings. Observations over longer periods of time (or from different locations) together with the use of a suitable model of the world will enable the observer to realize the "objective reality" of the gravitational lens configuration. However, this does not change anything about her subjective perceptions of the instantaneous local effects. 
In principle, light can travel in both directions on the curved paths between S and A depicted in Fig. 4. Thus, $\mathrm{S}$ also "sees" three images of A and perceives three corresponding contributions to the gravitational field strength. The gravitational lens induces an enhancement of the mutual interaction between $\mathrm{S}$ and $\mathrm{A}$. We have to keep in mind that the static configuration of star, gravitational lens and observer implied here is an idealization. In reality the exact reversibility of the light travel is not given. Naturally the objects are in the state of free fall and are moving relative to each other. The basic rationale is not substantially affected by this fact, quite the contrary: the key role of the instantaneous local effects on an observer is even more pronounced.

\section{Relevance of the GLG for astrophysics}

In General Relativity Newton's law of gravity still is a good approximation for the weak gravitational field at large distances from a single mass in empty space ("Newtonian limit"). The Schwarzschild solution of the Einstein field equations for a homogenous uncharged non-rotating sphere is just made that way [10] - and is even valid for Black Holes! Since a single (point) mass cannot transversally curve the field lines originating from itself, the "Gravitationally Lensed Gravitation" cannot emerge there.

In the case of several masses arranged separately from each other, Newton's theory of gravity states that the total gravitational field is the linear superposition of the undisturbed individual gravitational fields of all the (point) masses. At any location the vectorial sum of the unchanged gravitational field strength contributions of these masses has to be calculated.

But after our new insights we here have to consider the GLG: The gravitational field of each mass is distorted by the collective gravitational lens effect of all other masses. So, all individual gravitational field contributions are distorted and self-consistently merged into the resultant total field. The gravitation is both effecting spacetime curvature and being affected by it concurrently. In General Relativity it is already well known that the gravitation shows a strong nonlinear behavior, which makes the computation of strong field interactions of closely neighboring compact masses extremely complicated. The new insight here is, that due to the GLG strong effects may occur also in the weak field case of widely separated masses. Thus, the previously used approximation by Newton's law of gravity is not sufficient in such cases.

In the configuration of the Gravitational Lens Gedankenexperiment described above we have a straightforward situation, since the roles are clearly distinguishable. The weak gravitational field of our star does virtually not alter the gravitational field of the mass assembly which acts as gravitational lens here. In contrast to that, the gravitational lens leads to a substantial enhancement of the contribution of our star to the gravitational field at the location of observer A. The lens curves the field lines so that three gravitational fields reach the observer from slightly different directions and are superimposed just the same way as gravitational fields from different stars usually are.

The fact that gravitational effects of several celestial bodies superimpose and that every single celestial body causes an acceleration in the direction in which it is to be seen, has led to the discovery of the planet Neptune. This must hold true also in General Relativity since the physical results do not depend on the representation. According to the thought experiment the individual gravitational contributions of the "images" generated by the gravitational lens are superimposed at the observer in the same way.

This effect of enhancement of gravitational fields in directions where masses are arranged in line one after the other may result in stronger gravitational fields in the outer regions of extended disks of galaxies. So GLG may help to explain the rotation curves of galaxies. 
In the early universe inhomogeneities of mass density caused gravitational lensing. Concentrating and thinning-out of field lines could have occurred at different locations, far away from where weak curvatures of the field lines are induced by gravitational lensing. So GLG may explain a significant modulation of the gravitational field and thus help to understand the structure formation.

A direct measurement of the GLG effect would probably be difficult. Maybe the gravitational effect of a microlensing event in connection with a Jupiter transit before the Sun as seen from a space probe in the outer solar system could lead to a tiny change of its orbit. Indirect evidence from a characteristic shape of the gravitational lens of individual galaxies seems to be more promising. A gravitational field being stronger within the disk and weaker perpendicular to it would result in a lens shape being less spherical than that of a dominant halo of dark matter.

\section{Weakening of the gravitation due to cosmic expansion}

In all previous considerations it was implicitly assumed that stars, gravitational lenses and observers are not too far apart from each other, so that no significant redshift caused by cosmic expansion occurs. Now we will additionally take into account the effect of such a redshift.

We consider the emission of a light pulse consisting of a large number $N$ of photons of frequency $f$ and wavelength $\lambda$. Disregarding absorption and scattering, the number $N$ of photons does not change during the propagation over very large distances, but their wavelength $\lambda$ increases because of a redshift due to cosmic expansion. The energy $E$ of $N$ photons is given by:

$$
E=N \cdot h \cdot f=\frac{N \cdot h \cdot c}{\lambda}
$$

$h$ is Planck's constant. The energy of the photons depends on their frequency or wavelength and this applies to the exposure $H$ as well, since it is energy $E$ per area $A$ :

$$
H=\frac{E}{A}=h \cdot c \cdot \frac{N}{A} \cdot \frac{1}{\lambda}
$$

So, the exposure from a light pulse of a far distant event is depending on two factors: First, the number of photons per area $N / A$, which is determined by the paths of the photons from the source to the observer. Mostly the photons move away from each other on their diverging paths. Therefore, the number of photons per area usually decreases with increasing distance from the source. But occasionally the paths may be affected by gravitational lenses in a way that a concentration of the photons, namely an increase of its number per area, is induced. Second, the wavelength $\lambda$, which increases with the redshift caused by cosmic expansion and so becomes a position dependent property. Correspondingly, the cosmic expansion not only reduces the exposure but also weakens the gravitational field strength of the far distant object (see equation (10)). Since light and gravitation are affected by spacetime curvature in the same way, the gravitational field strength is reduced as much as the energy of the photons due to the redshift.

The position dependent quantities gravitational field strength $g$ and exposure $H$ (or intensity $I$ ) originating from the same source have the same ratio to each other everywhere, which is specific for that particular source. As we have seen above, a position dependency of the frequency $f$ or wavelength $\lambda$ of the photons may have to be considered. This is the case for a redshift due to cosmic expansion and, furthermore, for a redshift caused by strong gravitational fields likewise. As an example, for the Schwarzschild metric it can be easily confirmed that the locally measured gravitational field strength (or acceleration) and the locally measured intensity of radially ascending light show the same position dependency. 


\section{Conclusions}

The idea of focusing and enhancing gravitational fields may at first sound much like science fiction and remind one or the other of the tractor beam of the Starship Enterprise. Thus, the content of this work is about to join a motley collection of "paradoxes" of relativity theory: Thought experiments with results that seem implausible because they contradict our everyday experiences and previous convictions. But as with those "paradoxes", whose most prominent member is certainly the "Twin Paradox", the apparent contradictions are resolved in a thorough rational analysis, the initially unbelievable results follow logically compelling from the application of basic physical principles and finally replace our previous beliefs.

The application of Birkhoff's theorem of General Relativity to a star which undergoes a sudden mass loss by isotropic emission of a light burst leads to a useful connection between light and gravitation: The energy density passing by the observer causes a corresponding change of the gravitational field strength or acceleration. The local absolute change of the field strength has always and everywhere the same constant ratio to the local exposure from the light burst. This universally valid connection finally leads to the realization that in the case of a constant shining star its gravitational field strength has the same position dependency as its light intensity. This still applies in the presence of a gravitational lens which therefore affects the (weak) gravitational field just as the light: "Gravitationally Lensed Gravitation" (GLG). According to the "Gravitational Lens Gedankenexperiment" the total contribution of the star to the gravitational field strength at the observer is enhanced by the same factor as the total light intensity by all its images. The star's light rays arrive at the observer from several directions and so do its gravitational effects. The several images of the only star have the same effects on the observer as several individual stars ("seeing-is-believing"). The light makes visible the effect of the curved spacetime, which is not restricted to light but affects gravitation as well. Yet, its enhancement is not depending on the presence of light. The gravitational effect of a dark object is enhanced just in the same way.

The physics can be quite easily described within a field line model: A gravitational lens curves the field lines originating from a point source in the same way as it would curve the paths of photons emerging from the same point source. In such cases the assumption of a superposition of unaffected fields is obviously not valid. In connection with gravitational microlensing the GLG may lead to stronger gravitational effects on stars in the outer regions of extended galactic discs than would be expected according to Newton's law of gravity.

For a far distant object the cosmic expansion leads to a redshift and thus to a decrease of its light intensity. Similarly, its gravitational field is weakened. A concept of a model with light-analog "geodesic" field lines has to address this issue by adding an attribute similar to a frequency or wavelength. It can be shown that such a model correctly reproduces the position dependency of the locally measured gravitational acceleration in the Schwarzschild metric.

The method of presentation chosen here concentrates on the description of the essential physical relationships in a generally valid form, without getting lost in mathematical details of the representation of a specific coordinate system. Since the physical phenomena and processes itself do not depend on the model representation, the results of this work should also be reproducible by solving the Einstein field equation in the metric formalism. 
The finding of identical position dependencies of the gravitational field strength and the light intensity originating from the same object together with the knowledge of the effect of a gravitational lens on the light intensity enable us to derive the effect of a gravitational lens on the gravitational field of the object.

The "Gravitationally Lensed Gravitation" (GLG) provides an admittedly surprising but still simple and consistent description of the physical effects which are to be expected in the scenario of the Gravitational Lens Gedankenexperiment.

\section{Acknowledgement}

I would like to thank my parents for their support and the freedom they gave to my scientific curiosity. Many thanks are also due to my teachers for their enthusiasm and for laying solid foundations. I very much appreciate all those who strive for comprehensible presentation of scientific findings in books, journals and online, especially in the Wikipedia and on YouTube. Thanks to all who kindly answered my questions about General Relativity. Many thanks to all who helped with the proofreading and finishing touches to this paper. I would especially like to thank my wife for her support, patient encouragement and for her great forbearance when my thoughts once again were circling around galaxies billions of light years away.

\section{References}

[1] H. Hornung, "A solar eclipse sheds light on physics," 29 Mai 2019. [Online]. Available: https://www.mpg.de/9244824/solar-eclipse-1919.

[2] A. Einstein, "Lens-Like Action of a Star by the Deviation of Light in the Gravitational Field," Science, New Series, vol. 84, no. 2188, pp. 506-507, 4 Dezember 1936. PDF

[3] F. Zwicky, "Nebulae as gravitational lenses," Physical Review, vol. 51, no. 4, p. 290, 1937. PDF

[4] S. Refsdal, "On the possibility of determining Hubble's parameter and the masses of galaxies from the gravitational lens effect.," Monthly Notices of the Royal Astronomical Society, vol. 128, no. 4, pp. 307-310, 1964. PDF

[5] ESA/Hubble and NASA, "File:QSO B0957+0561.jpg," Wikimedia Commons, [Online]. Available: https://commons. wikimedia.org/w/index.php?curid=30719704.

[6] D. Walsh, R. F. Carswell and R. J. Weymann, "0957+ 561 A, B: twin quasistellar objects or gravitational lens?," Nature, vol. 279, no. 5712, pp. 381-384, 1979.

[7] Wikipedia, "Twin Quasar," [Online]. Available: https://en.wikipedia.org/wiki/Twin Quasar.

[8] P. Kelly, S. Rodney, T. Treu and M. Jäger, "Caught in the act: Hubble captures first-ever predicted exploding star," 1612 2015. [Online]. Available:

https://www.spacetelescope.org/news/heic1525/. 
[9] T. Padmanabhan, "Gravity bends electric field lines," in Sleeping Beauties in Theoretical Physics, Cham, Springer International Publishing Switzerland, 2015, pp. 279-292. PDF

[10] W. Rindler, Relativity: Special, General, and Cosmological, Oxford: Oxford University Press on Demand, 2006.

[11] C. de Rham, "Nature of the Graviton," TEDxCLESalon, [Online]. Available: https://youtu.be/ThQ8P1KSFiw?t=686.

[12] M. Kutschera, "Monopole gravitational waves from relativistic fireballs driving gamma-ray bursts," Monthly Notices of the Royal Astronomical Society, vol. 345, no. 1, pp. L1-L5, 17 Juli 2003. PDF

[13] D. Kodwani, U.-L. Pen and I.-S. Yang, "Supernova energy measurement with longitudinal gravitational memory effect," memory, vol. 2, p. 2, May 2016. PDF

[14] K. D. Olum, E. Pierce and X. Siemens, "Detectability of gravitational effects of supernova neutrino emission through pulsar timing," Physical Review D, vol. 88, no. 4, p. 043005, 2013. PDF

[15] U. Borgeest and S. Refsdal, "Der Gravitationslinseneffekt," Physikalische Blätter, vol. 40, no. 1, 1984. PDF

[16] M. A. Abramowicz, "Black holes and the centrifugal force paradox," Scientific American, vol. 268, no. 3, pp. 74-81, 1993.

[17] D. B. Haarsma, J. N. Winn, I. Shapiro and J. Lehár, "The central component of gravitational lens Q0957+ 561," The Astronomical Journal, vol. 135, no. 3, p. 984, 2008. PDF 